\title{
Outcomes Following Alonso-Llames Triceps-Sparing Approach for Type-C Distal Humerus Fractures: A Single Center Study
}

\author{
Wahyu Widodo, Andi Praja Wira Yudha Luthfi \\ Department of Orthopaedic Surgery, Faculty of Medicine Universitas Indonesia, Cipto Mangunkusumo Hospital, Jakarta, Indonesia.
}

\section{Corresponding Author: \\ Dr. Wahyu Widodo \\ Email: wawidfkui@yahoo.com}

This is an Open Access article distributed under the terms of the Creative Commons Attribution License (creativecommons.org/ licenses/by/3.0).

$\begin{array}{lll}\text { Received } & : & \text { December 1, 2018 } \\ \text { Accepted } & : & \text { April 5, 2019 } \\ \text { Published } & : & \text { April 20,2019 }\end{array}$

\begin{abstract}
Introduction: The elbow joint is a combination of stability and motion of three individual articulations. Disruption resulting from distal humerus fractures will affect the functions and motions of the elbow joints significantly. Adequate restoration of elbow function compels reconstruction of the anatomical articular surface and steady fixation of the fractured fragments for early and complete rehabilitation. Any disruption of the triceps from the olecranon leads to weakened extension power. Triceps-sparing techniques may be able to maintain elbow strength by keeping the olecranon-triceps unit intact. Methods: A retrospective study was completed in Cipto Mangunkusumo Hospital, Jakarta comprising five cases of inter-condylar fracture of the humerus. The fractures types were AO type $\mathrm{C} 1$ and $\mathrm{C} 2$ fractures of the distal humerus. Exposure of supra-condylar ridges on the distal humerus were achieved by raising the medial and lateral flaps as in Alonso-Llames triceps-sparing approach. Double plating was used in all cases to stabilize the fractures fragment. Results: All five fractures had achieved union at the fourth month of followup. None of the patients endured loss of supination or pronation though all patients had several degree of flexion/extension reductions compared with the normal side. The mean Mayo Elbow Performance Index (MEPI) was 94 (range 85 to 100) representing superb outcome in twelve months follow up. Conclusion: The usage of triceps-sparing method to uncover type $\mathrm{C}$ distal humerus fractures led to decreased post-operative complication rate and quicker patient recovery without reducing the view of the articular surface or disturbing fracture reduction during fixation.
\end{abstract}

Keywords: Elbow Joint, Fracture Fixation, Humerus, Olecranon Process, Retrospective Studies.

\section{Introduction}

The elbow joint is an exceptional combination of stability and motion of three individual articulations [1]. Disruption on intra-articular anatomy, will affect the functions and motions of the elbow joints significantly. These fractures continue to be difficult to deal with. The factors vital in managing distal humerus fractures are articular congruity restoration, secured bone fixation, attainment of bone healing, and preservation of a working range of motion $[2,3]$.

Inter-condylar fractures of the distal humerus (AO type C) are responsible for around 1\% of all adult fracture cases [4]. Adequate restoration of elbow function compels reconstruction of the anatomical articular surface and steady fixation of the fractured fragments in order to enable early and complete rehabilitation. Distal articular humerus fractures are rather managed with open reduction and internal fixation (ORIF). The surgery entails difficult techniques and ample exposure of the distal humerus articular surface [5]. Olecranon osteotomy is most often approach used for ORIF of intra-articular fractures of the distal humerus. This method exposes the articular surfaces of the elbow and distal humerus columns extensively. 
Drawbacks of this method are extended duration of the operation to conduct osteotomy and fixation procedure, and increased likelihood of restricted post-operative mobilization and physiotherapy $[1,2,6]$. Moreover, frequent complications are reported to be symptomatic malfunction of olecranon fixation devices which may have to be removed surgically, non-united olecranon, and elimination of osteotomy reduction. Any disruption of the triceps from the olecranon leads to weakened extension power. Tricepssparing techniques maintain elbow strength by keeping the olecranon-triceps unit intact $[3,7,8]$.

The present study aimed to demonstrate the functional outcome of the Alonso-Llames tricepssparing method for AO type-C distal humerus treated with ORIF. This study reviewed five cases of inter-condylar distal humerus fractures.

\section{Materials \& Methods}

A retrospective study was conducted in Cipto Mangunkusumo Hospital Jakarta comprising five cases of inter-condylar fracture of the humerus in 2016 and 2017. The fractures types were AO type C1and C2 inter-condylar fractures of the distal humerus [Fig.1]. These fractures endured internal fixation procedure with Alonso-Llames triceps-sparing method. The study consists of two cases of grade 1 compound fracture. One patient

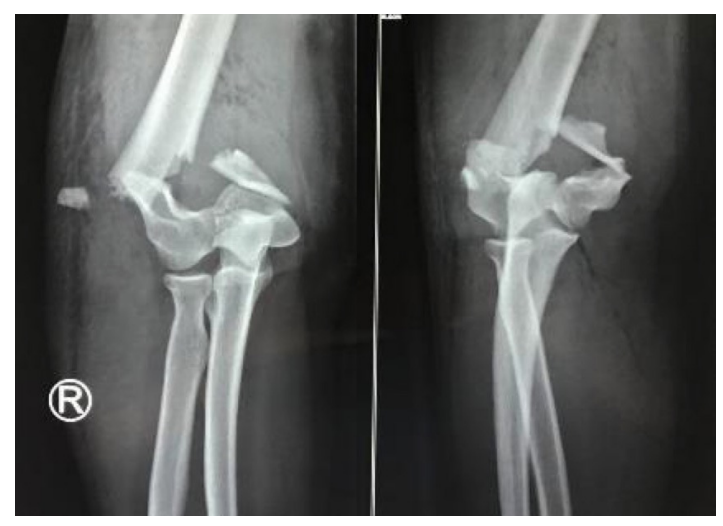

Fig.1: The anteroposterior and lateral radiographs showing the supracondylar comminution with intercondylar displacement. was male and four other patients were female. All patients suffered the injury as consequence of traffic accidents. Two of the patients had right elbow involvement and the remaining three had the left sided involvement. A 12-month follow up was conducted post-operatively on each patient.

All surgeries were completed under general anaesthesia and were positioned laterally with a pneumatic tourniquet on the upper arm. The arm was in relaxed position on top of pillows and the elbow was flexed at 90 to 100 degree. A straight incision was then made lateral to the olecranon tip [Fig.2]. Exposure of supra-condylar ridges on the distal humerus were achieved by raising the medial and lateral flaps. Isolation of the ulnar nerve was performed [Fig.3] through free dissection from posterior to the medial epicondyle distally approximately $7 \mathrm{~cm}$ to maintain the branches to flexor carpi ulnaris.

Reduction and fixation of the intraarticular constituent with $4.0 \mathrm{~mm}$ partially threaded cancellous screw were performed. The reconstituted condylar block was tentatively set to the medial and lateral columns using $1.8 \mathrm{~mm} \mathrm{~K}$

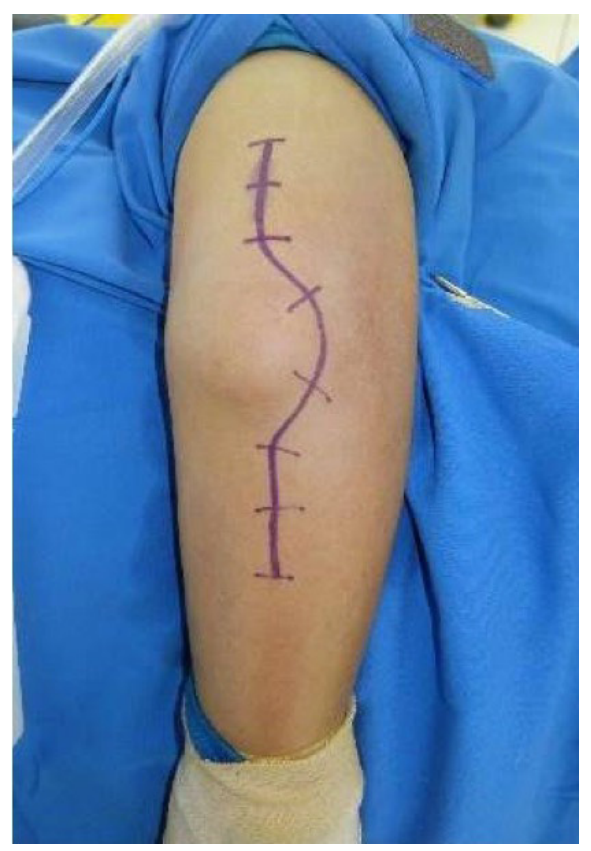

Fig.2: Posterior skin incision design. 
wires. Adjustment of two $3.5 \mathrm{~mm}$ reconstruction plates was conducted to fit the columns on the dorsal surface of the humerus. These plates were fixed using screws [Fig.4]. Anterior transposition of the ulnar nerve was done in all patients.

Immobilization of the extremities was applied for two weeks. Exercises of passive range of motion were initiated and supervised by a physiotherapist. Active-assisted elbow movements were introduced after one month and active elbow movements began after two months. Follow up was then continued by the physiotherapist for 4 to 6 months to increase range of motion. Anteroposterior and lateral $x$-rays [Fig.5] were obtained at three points of follow up at third, sixth and twelfth months. The range of motion was recorded in each follow up session and the strength of the triceps was recorded at the end of sixth and twelfth month.

\section{Results}

All five fractures had achieved union at the fourth month of follow-up. None of the patients endured loss of supination or pronation though all patients had several degree of flexion/extension reductions compared with the normal side. The overall arc of motion averaged at 115 degrees [Fig. 6]. The average range of motion was in between 0 and $20^{\circ}$ of extension (mean $5^{\circ}$ ) and between 120 and $140^{\circ}$ of flexion (mean $135^{\circ}$ ). Consistent with Medical Research Council (MRC) scale, one patient achieved a grade 4 in triceps and elbow flexion strength, and the other four patients obtained grade 5. All patients had secure elbows on the anteroposterior and medio-lateral planes at the end of the twelfth month follow up. All patients were able to carry out their daily tasks comfortably and unassisted at the end of the twelfth month, and none experienced pain which required analgesics. Infection did not develop in any of the patients. The mean Mayo Elbow Performance Index (MEPI) [9] was 94 (range 85 to 100) representing superb outcome.

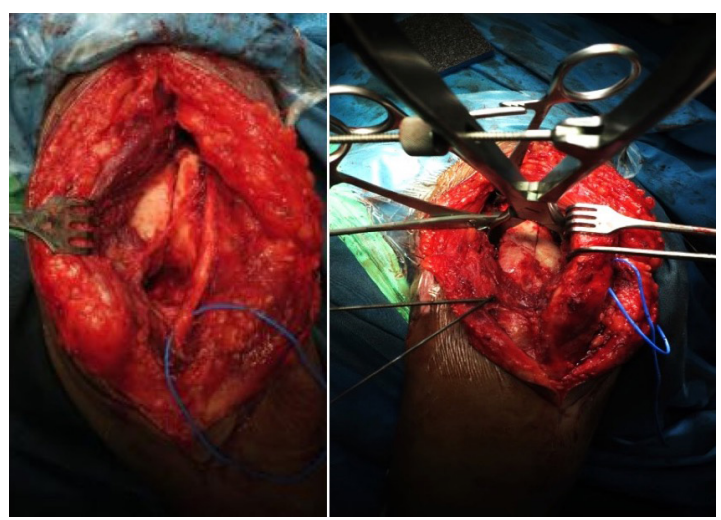

Fig.3: Medial and lateral flaps were raised to expose the supra-condylar ridges on either side of the distal humerus. Ulnar nerve was isolated using a silicone sling.

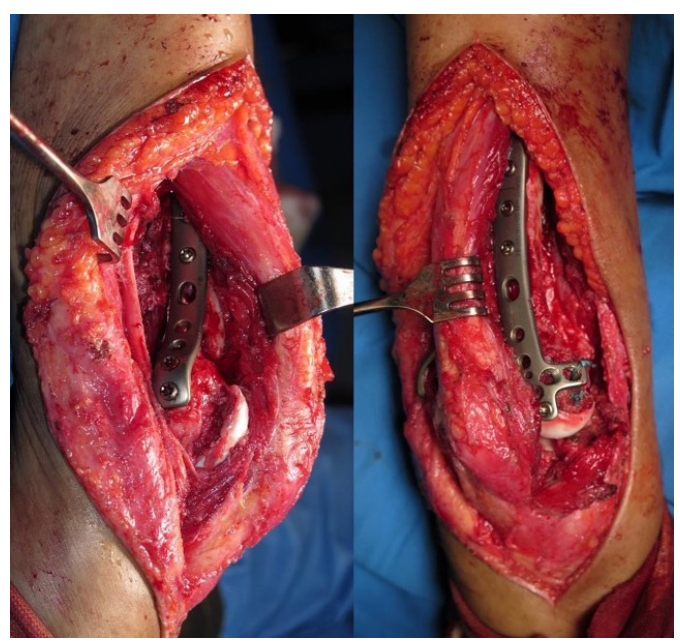

Fig.4: Fixation using plates on both column.

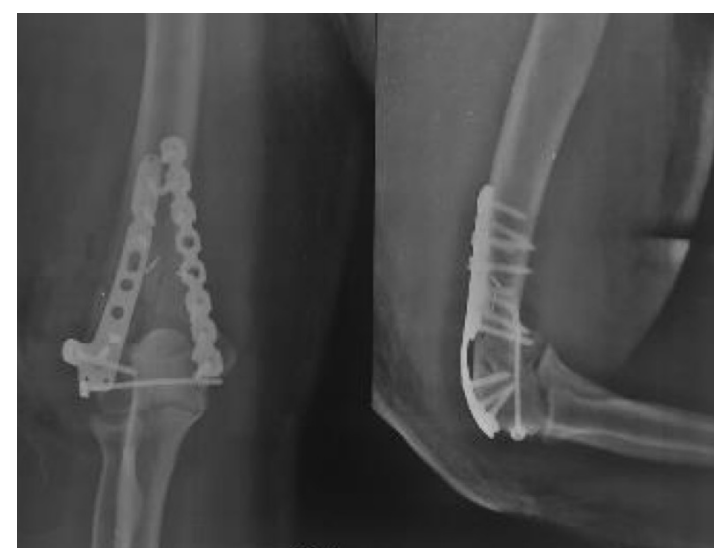

Fig.5: Post-operative radiographs at three weeks in anteroposterior and lateral view. The lateral plate could be placed distally and posterior despite intact extensor mechanism. 


\section{Discussion}

The clinical outcome of distal humerus fractures tends to rely more on the pattern of the injury and its ability to achieve anatomical reduction and secure fixation than on the applied surgical management [5]. Numerous surgical methods have been investigated, each with its own gains and losses [10]. While there is still no consensus addressing the optimal surgical method for open reduction and internal fixation as intra-articular distal humerus fractures management, many methods including triceps-splitting, triceps reflecting (TRAP), Bryan Morey's method and olecranon osteotomy are considered common posterior methods towards the elbow. Among these methods, chevron osteotomy of the olecranon is up until now still set to be the customary approach as it visualization of the distal humerus articular surface [11].

Reconstruction of osteotomy may cause delayed union, non-union, and bump of implant, which may compel further surgery. Correspondingly, triceps-splitting method does not produce adequate exposure of the articular surface compared with other methods; this method has one further drawback of direct muscle trauma, causing fibrosis and disruption to inter-muscular nerve branches, which can lead to muscle weakness [12]. Alonso-Llames first reported the paratricipital method in 1972 for pediatric supra-condylar fractures treatment [13]. As this method applies relatively bloodless plane and does not cause direct trauma to the triceps muscle, it may minimize formation of the scar and lessen post-operative triceps muscle dysfunction.

In our case series, we perform surgical treatment with paratricipital method on three patients with a type-C2 fracture and two patients with a type- $\mathrm{C} 1$ fracture. We did not find any substantial difficulties in conducting reduction of the fracture fragment and articular surface. After 12 months of post-operative follow up, overall elbow function evaluation resulted in a mean MEPI of 94

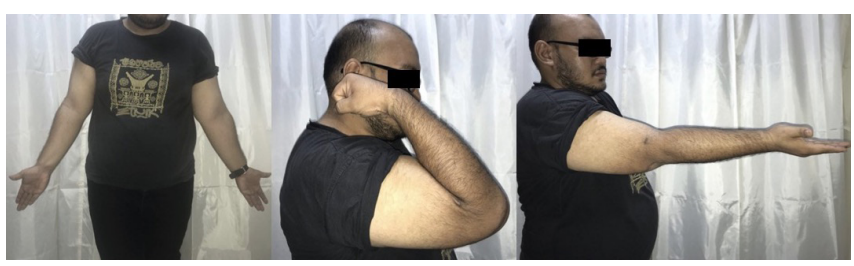

Fig.6: Twelve months after the operation, MEPI score of 100.

points. The average range of motion was in a range between $0-20^{\circ}$ of extension (mean $5^{\circ}$ ) and 120 $140^{\circ}$ of flexion (mean $135^{\circ}$ ). Based on the Medical Research Council (MRC) scale, one patient had a grade 4 in triceps and elbow flexion strength, and the other four had grade 5. All patients achieved elbows stability on the antero-posterior and mediolateral planes by the end of the twelfth month follow up. All subjects were able to could do their daily activities unassisted and comfortably by the end of the twelfth month, and none experienced pain needing analgesics. All of them had resumed their former activities.

Erpelding et al. [10] reported in their own series utilizing similar method, the mean DASH score of 15.9 points demonstrates mild impairment, which is similar with results of other studies with the DASH scores from 12 to 20 points. A mean MEPI score of 91.5 points was obtained in overall elbow function evaluation, which consists of fifteen excellent, seven good, two fair, and no poor grades. This value is consistent with the other studies. Gosal et al. reported their series of 23 patients with AO type-C fracture treated with the paratricipital method with 28 months of follow up. The study produced a mean MEPSI of 93 (range 72 to 100), exhibiting excellent outcome and all of the subjects had grade 5 in triceps strength and elbow stability on the antero-posterior and medio-lateral planes by the last point of follow up [11].

Based on our findings and experiences, we considered that the triceps-sparing method may be more advantageous than olecranon osteotomy in managing elderly patients with distal humerus fractures. In general, $\mathrm{C} 1$ and $\mathrm{C} 2$ fractures 
management with the triceps-sparing method leads to shorter operation time, decreased blood loss, lower complication rate, and improved elbow motion and function. These may be credited to a number of aspects: simpler exposure procedure which reduces trauma, substantially leads to shorter operation time and reduced blood loss and may have lowered wound infection risk and systematic post-operative complications. These aspects are problematic for elderly patients. Second, the tricepssparing method evades downsides of olecranon osteotomy. This enabled us to reassure patients to execute a full range of elbow motions without any worries regarding non-union of the osteotomy or loosening of the implantation. Alleviation of these worries allow patients to execute triceps exercises shortly subsequent to the operation and consequently did not endure much triceps strength loss [12]. Additionally, active and passive ROM exercises may have supported the prevention of triceps weakness and elbow stiffness. Ultimately, the greatest benefit of triceps-sparing method is its ability to provide surgeons with several options during the operation. The triceps-sparing method can be converted to an olecranon osteotomy without any difficulties when wider intra-articular exposure is needed for ORIF $[1,14,15]$.

Compared with olecranon osteotomy, the triceps-sparing method generally produced a lower post-operative complication rate and shorter time needed for rehabilitation, while still upholding the advantages of a wide exposure $[10,12,16]$. However, Chen et al. [4] showed an improved functional outcome for the olecranon osteotomy in 60-year-old patients and above. The study showed similar results in patients less than 40 years. Zhang et al. exhibited benefits of the sparing method in $\mathrm{AO}$ type $\mathrm{C} 1$ and $\mathrm{C} 2$ fractures but no substantial difference in $\mathrm{AO}$ type $\mathrm{C} 3$ fractures in comparison with the osteotomy method $[3,12,17,18]$. Paralleling the triceps-split method with the standard olecranon osteotomy, Meija et al. in 2008 exhibited no substantial difference in clinical outcome, verifying results from McKee et al. which previously showed similar functional results in both groups with loss of elbow extensor strength by around 25\% in both methods [19].

It has to be noted that this approach is not always applicable in any distal humeral fractures. The verdict to apply this method in complicated fracture types with significant intra-articular comminution, such as type-C3 fractures, should be created on a case-by-case basis following thorough review of imaging studies and deliberation of the surgeon's skills regarding the method. One of the benefits of this method is the relief acknowledging possible conversion to an olecranon osteotomy without further soft-tissue injury when the reduction cannot be achieved or sufficiently evaluated intraoperatively [10].

Study limitations consisted of small number of patients with only $\mathrm{AO}$ type $\mathrm{C} 1$ and $\mathrm{C} 2$ fractures and exclusion of patients in whom the approach was changed to an olecranon osteotomy. However, our goal was to determine a baseline for functional elbow outcomes and motion subsequent to a specific approach as follow-up data have been inadequate up to now. It is critical to consider this when paralleling elbow motion findings with earlier studies which comprised more aggressive methods with or without more complexed fracture patterns.

\section{Conclusion}

The usage of triceps-sparing method to uncover type $\mathrm{C}$ distal humerus fractures led to decreased post-operative complication rate and quicker patient recovery without reducing the view of the articular surface or disturbing fracture reduction during fixation. However, a prospective randomized controlled study with a larger population is needed to verify our findings. We consider that the learning curve is vital for triceps-sparing method, as it demands skills experience and technical training. 
Contributors: WW: manuscript editing, study conceptualization, patient management; APW, YL: manuscript writing, data collection, patient management. WW will act as guarantor. Both authors approved the final version of this manuscript.

Funding: None; Competing interests: None stated.

\section{References}

1. Gupta G, Ghosh S, Chaudhuri A, Datta S, Dutta S, Dugar N. Comparative study of different approaches for open reduction and internal fixation in fractures of distal humerus. Saudi J Sport Med. 2015;15:68-73.

2. Mühldorfer-Fodor M, Bekler H, Wolfe VM, McKean J, Rosenwasser MP. Paratricipital-triceps splitting \& quot; two-window \& quot; approach for distal humerus fractures. Tech Hand Up Extrem Surg. 2011;15:156-161.

3. Tirkey R, Jamdar J, Savant R. Prospective study of distal humerus fracture fixation with an extensor mechanism on approach. 2017;3:506-508.

4. Chen G, Liao Q, Luo W, Li K, Zhao Y, Zhong D. Triceps-sparing versus olecranon osteotomy for ORIF: Analysis of 67 cases of intercondylar fractures of the distal humerus. Injury. 2011;42:366-370.

5. Ziran BH, Smith WR, Balk ML, Manning CM, Agudelo JF. A true triceps-splitting approach for treatment of distal humerus fractures: A preliminary report. J Trauma Inj Infect Crit Care. 2005;58:70-75.

6. Mondal J, Krishna C, Ganguli R. Paratricepital approach for fixation of distal humerus fracture in adults - A good alternative. International Journal of Orthopaedics Sciences. 2017;3:526-533.

7. Illical EM, Farrell DJ, Siska PA, Evans AR, Gruen GS, Tarkin IS. Comparison of outcomes after triceps split versus sparing surgery for extra-articular distal humerus fractures. Injury. 2014;45:1545-1548.

8. Ilyas AM, Jupiter JB. Treatment of distal humerus fractures. Acta Chir Orthop Traumatol Cech. 2008;75:615.

9. Longo UG, Franceschi F, Loppini M, Maffulli N, Denaro V. Rating systems for evaluation of the elbow. Br Med Bull. 2008;87:131-161.
10. Erpelding JM, Mailander A, High R, Mormino MA, Fehringer EV. Outcomes following distal humeral fracture fixation with an extensor mechanism-on approach. J Bone Jt Surg - Ser A. 2012;94:548-553.

11. Gosal G, Singh M. A study to assess outcome of osteosynthesis of AO Type $\mathrm{C}$ fractures of distal humerus using triceps-on approach. International Journal of Science and Research. 2015;4:2013-2016.

12. Zhang C, Zhong B, Luo CF. Comparing approaches to expose type $\mathrm{C}$ fractures of the distal humerus for ORIF in elderly patients: Six years clinical experience with both the triceps-sparing approach and olecranon osteotomy. Arch Orthop Trauma Surg. 2014;134:803-811.

13. Alonso-Llames M. Bilaterotricipital approach to the elbow: Its application in the osteosynthesis of supracondylar fractures of the humerus in children. Acta Orthop. 1972;43:479-490.

14. Boorman RS, Page WT, Weldon EJ, Lippitt S, Matsen FA. A triceps-on approach to semi-constrained total elbow arthroplasty. Tech Shoulder Elb Surg. 2003;4:139144.

15. Trikha V, Goyal T. Intercondylar humerus fractures: Current concepts and controversies. J Clin Orthop Trauma. 2010;1:57-65.

16. Aktekin CN, Toprak A, Ozturk AM, Altay M, Ozkurt B, Tabak AY. Open reduction via posterior triceps sparing approach in comparison with closed treatment of posteromedial displaced Gartland type III supracondylar humerus fractures. J Pediatr Orthop Part B. 2008;17:171178.

17. Pollock JW, Athwal GS, Steinmann SP. Surgical exposures for distal humerus fractures: A review. Clin Anat. 2008;21:757-768.

18. Tak SR, Dar GN, Halwai MA, Kangoo KA, Mir BA. Outcome of olecranon osteotomy in the trans-olecranon approach of intra-articular fractures of the distal humerus. Ulus Travma ve Acil Cerrahi Derg. 2009;15:565-570.

19. Silva DM, Santos RMDL, Alberto M, Ramos C, Pérez CG. Functional results of two different surgical approaches in patients with distal humerus fractures type $\mathrm{C}$ (AO). Acta ortopédica Mex. 2008;22:26-30. 